\title{
The Production of Inactive Phage P22 Particles Following Induction'
}

\author{
VANCE ISRAEL ${ }^{2}$

\begin{abstract}
Department of Human Genetics and The Lawrence D. Buhl Center for Human Genetics, University of Michigan, 1133 E. Catherine St., Ann Arbor, Michigan 49104
\end{abstract} \\ Accepted June 28, 1967
}

\begin{abstract}
Using an in vitro system for tail attachment, it is demonstrated that following ultraviolet (UV) induction of Salmonella typhimurium LT2 (P22) most of the particles formed lack a functional tail. This effect is found at all temperatures studied, but is more pronounced at temperatures of $37^{\circ}$ and above. Similar results are described for thermal and spontaneous induction. Tailless particles are found following infection only at temperatures of $42^{\circ}$ and above. Temperature pulse experiments on UV-induced lysogens suggest that late in the growth cycle tail synthesis is inhibited by high temperature. There is also an earlier temperature-sensitive step, which probably causes the production of inactive heads at $40^{\circ}$.
\end{abstract}

\section{INTRODUCTION}

Lytic infections of Salmomella typhimurium with phage P22 result in burst sizes ranging from 200 to 500 (Levine, 1957), but UV inductions of lysogens yield bursts of this size only when carried out at temperatures below $37^{\circ}$. At $40^{\circ}$ the burst size is often less than one. This has recently been shown to be due in part to the formation of heads which lack a complete base plate, or tail. These particles can be activated by the addition of high concentrations of tail parts, spindle-shaped protein molecules which assume their characteristic sixsided configuration only in the presence of heads (Israel et al., 1967).

The present communication reports studies on phage P22 production following induction using the in vitro system of tail attachment to assay "active" heads which lack a complete tail.

${ }^{1}$ Submitted in partial fulfillment of the requirements for the degree of doctor of philosophy, Horace H. Rackham School of Graduate Studies, University of Michigan, Ann Arbor.

2 Present address: Graduate Department of Biochemistry, Brandeis University, Waltham, Massachusetts.

\section{MATERIALS AND METHODS}

Phage and bacterial strains. Salmonella typhimurium LT2 was used for all infections and as background for plating. The phage P22 lysogens LT2 $(c+)$ (Levine, 1957) and LT2 ( $\left.t s c_{2}\right)$ (Israel et al., 1967) have been previously described, as have the phage P22 strains $c+$ and $t s c_{2}$ (Levine and Smith, 1964).

Media. Buffered saline (BS), nutrient agar, soft agar for top layers (Levine, 1957), and supplemented M9 (Smith and Levine, 1964) have all been previously described.

Definitions. An active phage is defined as a plaque-forming unit (PFU). An active head is any particle which can form a plaque after being exposed to tail parts. Thus active head titer is always greater than or equal to active phage titer. Inactive heads cannot form plaques when provided with a functional tail. Active head titer plus inactive head titer is equivalent to phage particle titer. One tail is defined as the number of tail parts required to saturate one head.

Active head assay. The reaction mixture for the assay of active heads consisted of (1) $10^{10}$ purified tails (Israel et al. 1967), (2) an appropriately diluted sample of 
heads (below $10^{9}$ heads $/ \mathrm{ml}$ ), and (3) BS in a final volume of $1 \mathrm{ml}$. The reaction was allowed to proceed for several hours at room temperature, which is more than adequate to ensure completion.

$U V$ inductions. LT2 $(c+)$ was grown at $37^{\circ}$ with aeration in supplemented M9 to a concentration of $10^{8}$ cells $/ \mathrm{ml}$. The culture was then transferred to a petri dish and exposed to UV light for 20 seconds (15-Watt GE germicidal lamp at $50 \mathrm{~cm}$ ). For the temperature range experiment the induced culture was then diluted to a concentration of $2 \times 10^{3}$ cells $/ \mathrm{ml}$ in supplemented M9 prewarmed to the appropriate temperature. When lysis was complete each culture was treated with chloroform and assayed for active heads and phage. In the case of the temperature pulse experiment, the culture was diluted 500-fold immediately after irradiation. At 5-minute intervals, an aliquot was diluted 10 -fold into $30^{\circ}$ media and after 9 minutes was returned to $40^{\circ}$ media by another 10-fold dilution. At 100 minutes after induction, all tubes were treated with chloroform and assayed for active phage and heads.

Thermal inductions. LT2 ( $\left.t s c_{2}\right)$ was grown in supplemented M9 with aeration at $30^{\circ}$ to a concentration of $10^{8}$ cells $/ \mathrm{ml}$, sedimented, and washed three times with BS to lower the background of free phage. The washed cells were then resuspended at a concentration of $2 \times 10^{3}$ cells $/ \mathrm{ml}$ in supplemented M9 prewarmed to $40^{\circ}$. At various times throughout the cycle an aliquot was chilled, assayed for infective centers, and then placed at $25^{\circ}$. When lysis was complete each aliquot was treated with chloroform and assayed for active phage and héads.

Infections. Log phase cells grown in supplemented M9 were sedimented, washed twice with sterile water, and resuspended in $\mathrm{BS}$ at a concentration of $3 \times 10^{8}$ cells/ ml. Aeration was then resumed for 40 minutes at $37^{\circ}$ before the culture was infected with either $c+$ or $t s c_{2}$ phage at a multiplicity of two phage per cell. After a 5 -minute adsorption period the cells wcre assayed for infective centers and diluted to a concentration of $2 \times 10^{3}$ cells $/ \mathrm{ml}$ in sup- plemented M9 prewarmed to the appropriate temperature.

\section{RESULTS}

\section{UV Induction}

Active phage production following UV irradiation of LT2 $(c+)$ is markedly temperature dependent. From a high of 200 at $28^{\circ}$, the burst size drops to approximately 3 at just under $40^{\circ}$ (Fig. 1, curve $A$ ). The burst size of active heads, however, is about 1000 at $28^{\circ}$ and 200 at $40^{\circ}$ (Fig. 1, curve $B$ ).

Three conclusions can be drawn from these experiments: (1) UV induction at any temperature results in the production of many more active heads than active phage. (2) This effect is exaggerated at higher temperatures; thus at $40^{\circ}$ active head titer is $\mathbf{1 0 0}$ times the active phage titer, while at $28^{\circ}$ it is only 5 times the active phage titer.

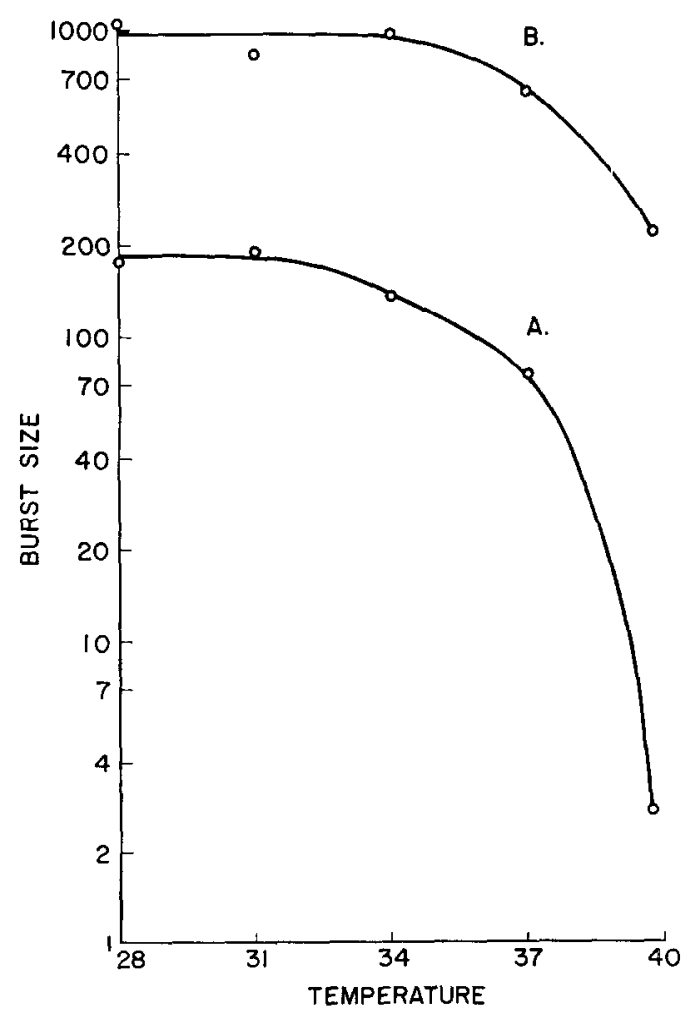

FIG. 1. Temperature dependence of burst size following UV induction of $\mathrm{LT}_{2}(c+)$. Curve $A$, active phage; Curve $B$, active heads. 


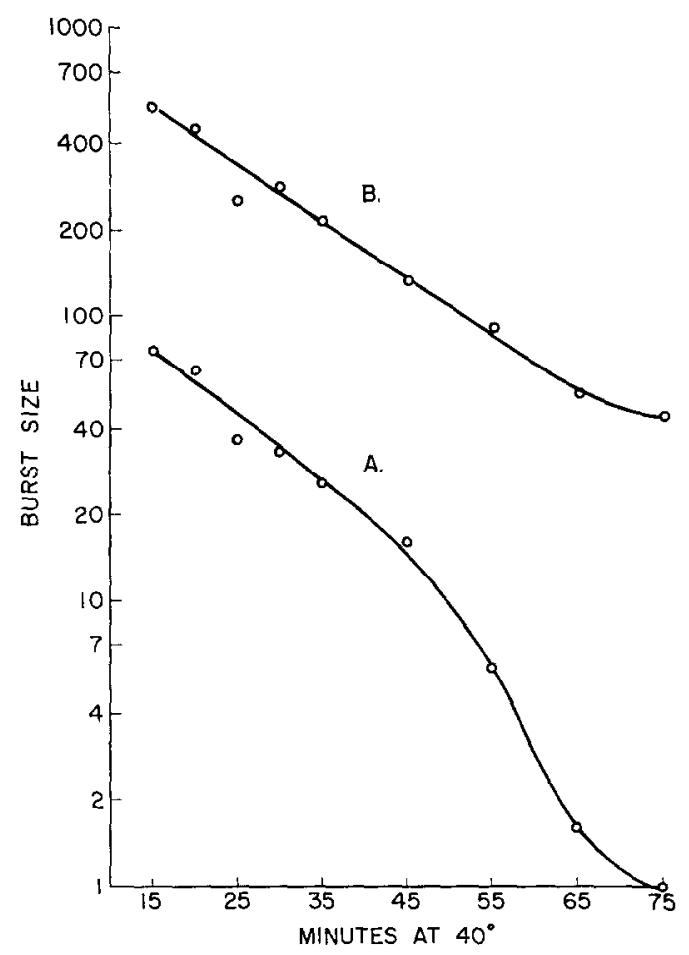

Fig. 2. Dependence of burst size on length of exposure to $40^{\circ}$ following thermal induction of LT2 ( $\left.t s c_{2}\right)$. Curve $A$, active phage; curve $B$, active heads.

(3) There is at least one other temperaturesensitive step after UV induction since active head titer is also decreased at higher temperatures.

\section{Heat Induction}

Analogous experiments were performed with a heat-inducible system. The burst size of active phage falls off with increasing exposure to $40^{\circ}$ from a high of 75 to a low of 1 (Fig, 2, curve $A$ ). Active head titer is higher in all cases, but still decreases significantly as a function of time at $40^{\circ}$ (Fig. 2, curve $B$ ).

Thus the three conclusions drawn for UV induction also hold for heat induction when length of exposure to $40^{\circ}$ is the variable as opposed to temperature of incubation. There are more active heads then active phage produced at all exposures; increasing exposure exaggerates this effect; and increasing exposure results in decreased active head production.

\section{Spontaneous Induction}

Similar results have been obtained for spontaneous induction. The free active phage titer of a culture of LT2 $(c+)$ grown at $27^{\circ}$ was found to be $10^{4} \mathrm{PFU} / \mathrm{ml}$ while the titer of active heads was 100 times this value. 'Thus even without artificial methods of induction, many more active heads than active phage are produced.

\section{Infection}

When infections are carried out under conditions similar to those employed in the induction experiments described above, there is no difference between active head and active phage titer (Fig. 3). However, at $42^{\circ}$ active head titer is much higher than active phage titer (Table 1). This has been found to be true in the case of $c+$ and $c_{1}$ phages as well as $t s c_{2}$.

w
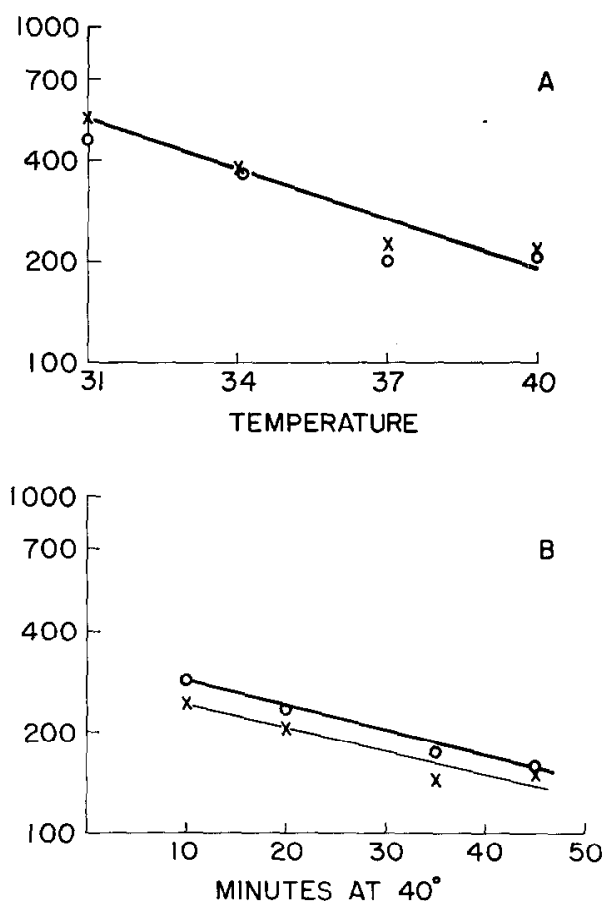

Frg. 3. (A) Temperature dependence of burst size following infection with $c+$ phage. $O--O$ represents active phage, $X-X$ represents active heads. (B) Dependence of burst size on length of exposure to $40^{\circ}$ after infection with $t s c_{2}$ phage. $O-O$ represents active phage, $X-X$ represents active heads. 
TABLE 1

Titers of Active Phage and Heads following Infection with Phage tsc at Various TEMPERATURES ${ }^{a}$

\begin{tabular}{lcccc}
\hline Temperature & $37^{\circ}$ & $40^{\circ}$ & $42^{\circ}$ & $44^{\circ}$ \\
Active phage titer & $4.14 \times 10^{10}$ & $1.19 \times 10^{11}$ & $5.7 \times 10^{8}$ & $3.6 \times 10^{6}$ \\
Active head titer & $3.89 \times 10^{10}$ & $1.13 \times 10^{11}$ & $4.34 \times 10^{10}$ & $6.42 \times 10^{8}$ \\
Active heads/active phage & 0.94 & 0.95 & 73 & 178 \\
\hline
\end{tabular}

${ }^{a}$ LT2 was grown to concentration of approximately $3 \times 10^{7}$ cells $/ \mathrm{ml}$ at $37^{\circ}$, then transferred to the appropriate temperature. Cultures were infected at a multiplicity of 10 phage/cell when cell titer was approximately $10^{8}$ cells $/ \mathrm{ml}$.

\section{Temperature Pulse Experiments}

Temperature pulse experiments were performed following UV induction to determine the timing of the temperature-sensitive steps. Following induction at $40^{\circ}$, aliquots were exposed to $30^{\circ}$ (cold pulsed) for 9 minutes at 5-minute intervals and then returned to $40^{\circ}$ to finish the growth cycle. A plot of the titers of active phage against time of pulse results in a biphasic curve (Fig. 1, curve A). The time of the initiation of DNA synthesis falls in the middle of the first peak. Active head titer (Fig. 4, curve $B$ ) is higher than active phage titer in all cases by at least a factor of ten, and the curve is monophasic.

The early peak of curve $A$ occurs at the same time as the peak of curve $B$. But curve $B$, which can be thought of as curve $A$ plus tail parts, lacks a late peak. Therefore, the late peak of curve $A$ must be the result either of increased tail synthesis at $30^{\circ}$, or of more efficient attachment of tail parts to heads.

\section{Particle Formation at High and Low Tem- perature}

The question remains whether the early temperature-sensitive step acts by causing a decrease in the number of particles made at high temperature or whether it causes the formation of inactive heads. $A$ partial answer to this question was obtained by comparing the $\mathrm{OD}_{260} /$ active head ratio of $\mathrm{CsCl}$ purified phage preparations which were produced at high and low temperatures. (Optical density at $260 \mathrm{~m} \mu$ is assumed to he proportional to particle titer.) For a preparation made by $\mathrm{UV}$ induction at $27^{\circ}$, the ratio was $1.54 \times 10^{-12}$ OD unit/active head; for one made at $40^{\circ}$ it was $4.55 \times$ $10^{-12}$ OD unit/active head. Since the particle to head ratio is three times higher for the preparation made at $40^{\circ}$, it is clear that

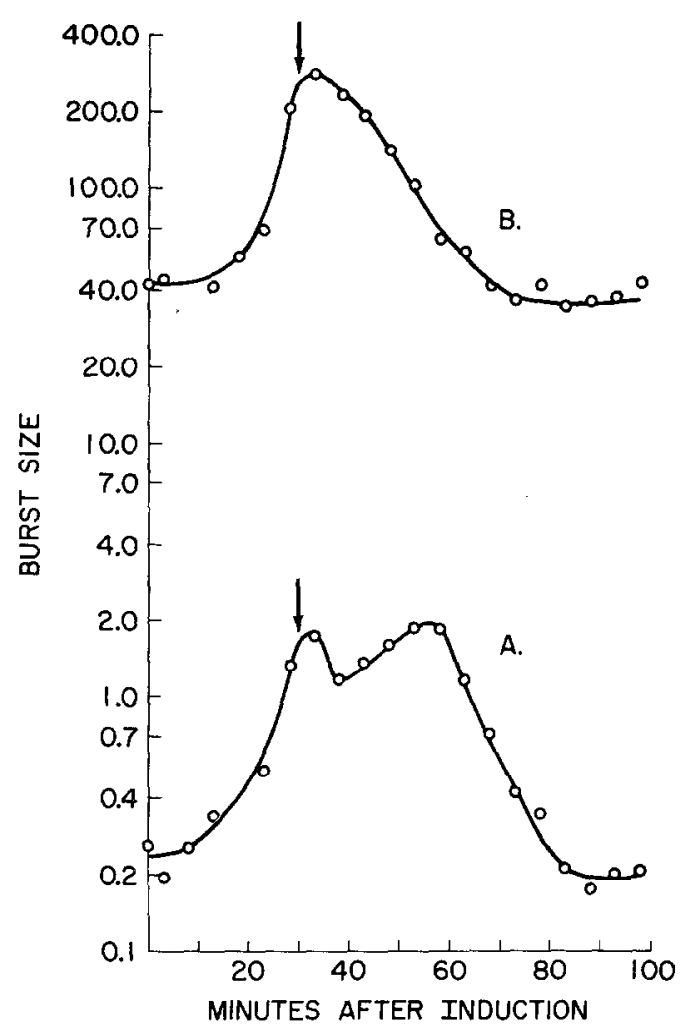

FIG. 4. Dependence of burst size on time of cold pulse. UV induction was carried out at $40^{\circ}$. Each circle represents the midpoint of a 9 -minute incubation period of $30^{\circ}$. However, the circles are plotted 3 minutes after the initiation of each pulse since the generation time is 100 minutes at $40^{\circ}$ and 150 minutes at $30^{\circ}$. The arrows represent the point at which DNA synthesis was initiated as determined by pulse labelling with thymidine ${ }^{3} \mathrm{H}$ (Smith and Levine, 1964). Curve $A$, active phage; curve $B$, active heads. 
high temperature causes the formation of inactive heads.

\section{DISCUSSION}

It has been noted by several people who have worked with P22 that yields of infectious phage are lower following induction than infection (Smith, Levine, Yamamoto, and Anderson, personal communications). This observation is confirmed and partially explained by the results presented here. These findings show that there are two heat-sensitive steps following induction and that induction itself results in the production of more active heads than active phage.

The early temperature-sensitive step (Fig. 4) may be identical to the $L$ function described by Smith (1967; Smith and Levine, 1967). Phage possessing the $L$ mutation behave normally on infection but cannot integrate into the bacterial chromosome as prophage. If they are integrated by complementation with a $c_{2}$ phage, the resulting lysogens produce large numbers of aberrant particles following UV induction. These defective particles, known as $L$ particles, possess host, as well as phage, DNA. The timing of the $L$ function is similar to the timing of the early step reported here. Thus, it is possible that the wild-type $L$ gene is somewhat temperature sensitive, and that the inactive heads detected after high temperature induction are actually $L$ particles.

There are two ways to explain the findings that induction causes more active heads to bc made and that high temperatures exaggerate this effect. Either not enough tail parts are made to accommodate all the heads, or enough tails are made, but for some reason they are prevented from reacting with heads. Although it cannot be rigorously excluded, the possibility that enough tails are made but do not attach is cxtrcmely unlikcly. The in vitro work shows that when either reactant is present in a concentration of around $10^{10} / \mathrm{ml}$, the reaction proceeds to completion in less than 1 hour over a wide range of temperatures, $\mathrm{pH}$, and salt concentrations (Israel et al., 1967; Israel, unpublished results). Thus, at intracellular concentrations, the reaction must be extremely rapid, and one would have to postulate either an extraordinarily strong specific inhibitor, or else severe compartmentalization of the two species. In the absence of any evidence to the contrary, insufficient tail synthesis seems much the more reasonable hypothesis.

Whatever the cause of the apparent tail deficiency, it is important to emphasize one other result of the in vitro study. This is the observation that if one starts with an equal number of heads and tails, halving the

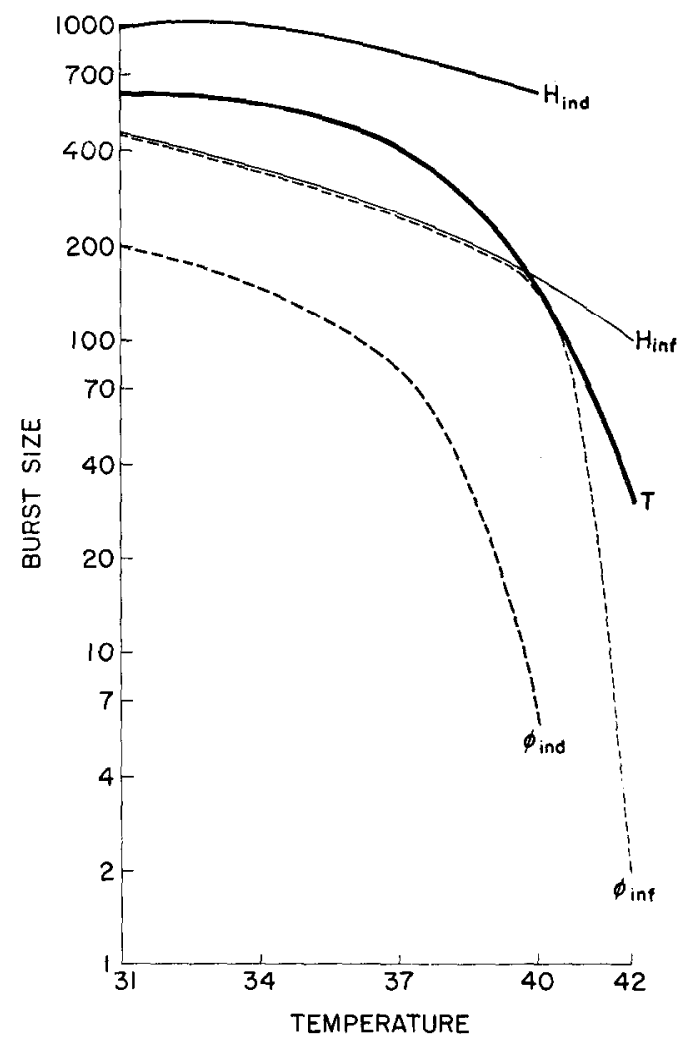

FIg. 5. Theoretical temperature dependence of head and tail synthesis. These curves demonstrate how the difference in temperature sensitivity of phage production following induction and infection can be reconciled with tail synthesis which is equally temperature-sensitive in both cases. Although consistent with observed data, the curves are theoretical, not empirical, and are in agreement with the formula given in the text. For the sake of clarity it is assumed that all heads are active. $\mathrm{H}_{\text {ind }}$ and $\mathrm{H}_{\text {inf }}$ refer to heads formed after induction and infection, respectively. $\phi_{\text {ind }}$ and $\phi_{\text {inf }}$ refer to phage formed after induction and infection, respectively. $T$ refers to tail synthesis for both cases. 
number of tails causes a tenfold reduction in the number of active phage produced, according to the formula: $\log \phi=3.3 \log$ $\mathrm{T}-2.3 \log \mathrm{H}$, where $\phi=$ phage, $\mathrm{H}=$ heads, $\mathrm{T}=$ tails, and $\mathrm{T} \leq \mathrm{H}$ (Israel, et al., 1967). Thus the tail deficiencies are not as dramatic as they first appear. For instance, if the burst size of phage is 10 and the burst size of active heads is 1000 , tail parts sufficient to saturate 250 heads have actually been made and attached. Furthermore, the discrepancy between active heads and active phage seen after low temperature induction can be explained either by inhibited tail synthesis (or attachment) or excess head synthesis. Since low temperature induction generally results in high burst sizes of active heads, the latter hypothesis is a reasonable one.

However, increased head synthesis is almost certainly not the explanation for the fact that active phage titer drops more rapidly than active head titer as the temperature of induction is increased. If tail synthesis were constant, almost an 8-fold increase in particles would be necessary to account for the 100 -fold drop in active phage titer observed in Fig. 1, curve $A$. This would mean that at $40^{\circ}$ the ratio of particles to active heads would be on the order of 40 . In fact, as shown above, it is approximately three.

One way to account for the sharp drop in active phage titer when the temperature of induction is increased is to postulate a naturally occurring temperature-sensitive gene for tail protein. But if this is the explanation, why should such a gene be more sensitive following induction than following infection? It may well be that this apparent difference in sensitivity to heat is an illusion. If we suppose that tail synthesis following infection is quantitatively identical at all temperatures to that following induction, it is only necessary to postulate less head than tail synthesis following infection at low tempcratures to account for the facts (Fig. 5). Then tail synthesis can be inhibited a certain amount before active phage titer will be affected. Following induction, on the other hand, where heads are always in excess, any inhibition of tail synthesis will be dramatically manifested in decreased phage titer. Under this scheme, the only difference between induction and infection (besides the early step causing inactive head formation) is that induction causes a stimulation of head synthesis compared to infection. This idea is supported by the preliminary finding that following a phage $c_{1}$ infection at $34^{\circ}$ there is approximately a $50 \%$ excess of tails formed.

In summary, the early temperature-sensitive step may prove to be identical to the $\mathrm{L}$ function, and tail synthesis may be as temperature sensitive following infection as it is following induction. If so, the only difference between infection and induction revealed by these studies is the fact that following induction there are always more active heads than active phage made. This is clearly not the case following infection, where active head and phage production are equal, except at very elevated temperatures. Why such a difference should exist is difficult to understand at present, but must be accounted for by any theoretical scheme which attempts to explain the induction of P22.

\section{ACKNOWLEDGMENTS}

This work was supported in part by predoctoral grant NIH-5-TO1-GM 0071-09. I wish to thank Dr. H. O. Smith for his valuable suggestions and criticisms during the course of this work and the preparation of the manuscript.

\section{REFERENCES}

Israel, J. V., Anderson, T. F., and Levine, M. L. (1967). In vitro morphogenesis of phage P22 from heards and hase plate parts. Proc. Natl. Acad. Sci. U.S., 57, 284-291.

Levine, M. (1957). Mutation in the temperate phage P22 and lysogeny in Salmonella. Virology 3, 22-41.

Levine, M., and Smith, H. O. (1964). Sequential gene action in the establishment of lysogeny. Science 146, 1581-1582.

Sмiтh, H. O. (1967). Production of defective particles by induction of Iysogens of integration deficient phage P22 mutants. Bacteriol. Proc. Am. Soc. Microbiol., p. 161.

SMrth, H. O., and Levine, M. (1964). Two sequential repressions of DNA synthesis in the establishment of lysogeny by phage P22 and its mutants. Proc. Natl. Acad. Sci. U.S. 52, 356363.

Smith, H. O., and Levine, M. (1967) A phage P22 gene controlling integration of prophage Virology 31, 207-216. 